\title{
HD 178892 - a cool Ap star with extremely strong magnetic field
}

\author{
T. Ryabchikova ${ }^{1,2}$, O. Kochukhov ${ }^{3}$, D. Kudryavtsev ${ }^{4}$, I. Romanyuk ${ }^{4}$, E. Semenko ${ }^{4}$, S. Bagnulo ${ }^{5}$, \\ G. Lo Curto ${ }^{5}$, P. North ${ }^{6}$, and M. Sachkov ${ }^{2}$
}

\author{
${ }^{1}$ Institute for Astronomy, University of Vienna, Türkenschanzstrasse 17, 1180 Wien, Austria \\ e-mail: ryabchik@inasan.ru \\ 2 Institute of Astronomy, Russian Academy of Sciences, Pyatnitskaya 48, 109017 Moscow, Russia \\ 3 Department of Astronomy and Space Physics, Uppsala University Box 515, 75120 Uppsala, Sweden \\ 4 Special Astrophysical Observatory of RAS, Nizhnij Arkhyz 369167, Russia \\ 5 European Southern Observatory, Casilla 19001, Santiago 19, Chile \\ ${ }^{6}$ Laboratoire d'Astrophysique, École Polytechnique Fédérale de Lausanne (EPFL), Observatoire, 1290 Sauverny, Switzerland
}

Received 25 October 2005 / Accepted 29 November 2005

\section{ABSTRACT}

We report a discovery of the Zeeman resolved spectral lines, corresponding to the extremely large magnetic field modulus $\left\langle B_{\mathrm{s}}\right\rangle=17.5 \mathrm{kG}$, in the cool Ap star HD 178892. The mean longitudinal field of this star reaches $7.5 \mathrm{kG}$, and its rotational modulation implies the strength of the dipolar magnetic component $B_{\mathrm{p}} \geq 23 \mathrm{kG}$. We have revised rotation period of the star using the All Sky Automated Survey photometry and determined $P=8.2478 \mathrm{~d}$. Rotation phases of the magnetic and photometric maxima of the star coincide with each other. We obtained Geneva photometric observation of HD 178892 and estimated $T_{\text {eff }}=7700 \pm 250 \mathrm{~K}$ using photometry and the hydrogen Balmer lines. Preliminary abundance analysis reveals abundance pattern typical of rapidly oscillating Ap stars.

Key words. stars: magnetic fields - stars: chemically peculiar - stars: individual: HD 178892

\section{Introduction}

About fifty years have passed since the discovery of the Zeeman resolved line profiles in intensity spectrum of a magnetic chemically peculiar star (Babcock 1960). Currently, 47 such objects, with magnetic field strength in the range of 2.8-33.5 kG, are known (Hubrig et al. 2005). The majority of the less massive (late A) strongly magnetic stars are slow rotators, with rotation periods of months or years. However, an exceedingly strong (up to $\sim 8 \mathrm{kG}$ ) longitudinal magnetic field, variable with a high amplitude and a period of about 8.3 days, was recently discovered in the poorly studied $\mathrm{SrCrEu}$ Ap star HD 178892 (BD+14 3811, HIP 94155) by El'kin et al. (2003) and Kudryavtsev et al. (2004). Such a strong field in combination with a relatively fast rotation is not known for any other low-mass Ap star, thereby making HD 178892 a very interesting target for in-depth analysis of the atmospheric structure and magnetic field geometry.

The lack of information about HD 178892 motivated us to obtain new magnetic and photometric measurements, and to acquire the first high-resolution spectroscopic observations for this star. With these new data, Zeeman resolved lines indicating a field modulus $\left\langle B_{\mathrm{s}}\right\rangle=17.5 \mathrm{kG}$ are detected in the spectrum of HD 178892, which confirms the presence of an extremely strong field in this star. We describe new observations of HD 178892 in Sect. 2 and determine fundamental stellar parameters in Sect. 3. The stellar rotation period and magnetic field are discussed in Sect. 4. Section 5 reports preliminary abundance analysis results, and conclusions are given in Sect. 6.

\section{Observations and data reduction}

El'kin et al. (2003) have selected HD 178892 as a candidate for magnetic measurements on the basis of low-resolution spectral observations of the $\lambda 5200 \AA$ depression profile. As described by El'kin et al., this low-resolution spectroscopy serves as an analogue of the $\Delta a$ photometry and helps to identify strongly magnetic cool Ap stars. Circular polarization analyzers (Najdenov \& Chuntonov 1976; Chuntonov 1997) attached to the Main Stellar spectrograph (MSS) of the 6-m telescope of SAO RAS were used to obtain 18 spectra of HD 178892. For 16 spectra, the $2 \mathrm{~K} \times 2 \mathrm{~K}$ pixel $\mathrm{CCD}$ detector was used, and the instrument was configured to give a spectral resolution $R=15000$ and a $240 \AA$ wavelength coverage in the $\lambda 4500 \AA$ region. Two additional MSS circular polarization observations were obtained for the $\mathrm{H} \alpha$ region. Polarized spectra were analysed with the help of ESO MIDAS, using our codes for Zeeman spectra reduction and $\left\langle B_{\mathrm{z}}\right\rangle$ measurements (Kudryavtsev 2000). The longitudinal component of the stellar magnetic field, $\left\langle B_{\mathrm{z}}\right\rangle$, 
Table 1. Spectroscopic and spectropolarimetric observations of HD 178892

\begin{tabular}{|c|c|c|c|c|}
\hline \multicolumn{5}{|c|}{ Circular polarization spectra } \\
\hline HJD & $\left\langle B_{\mathrm{z}}\right\rangle(\mathrm{G})$ & $S / N$ & $\lambda_{\mathrm{c}}(\AA)$ & Instrument \\
\hline 2452459.461 & $5940 \pm 520$ & 140 & 4500 & MSS \\
\hline 2452625.140 & $7200 \pm 380$ & 120 & 4500 & MSS \\
\hline 2452626.139 & $7380 \pm 380$ & 100 & 4500 & MSS \\
\hline 2452660.652 & $4400 \pm 710$ & 50 & 4500 & MSS \\
\hline 2452661.645 & $3620 \pm 700$ & 50 & 4500 & MSS \\
\hline 2452688.614 & $2160 \pm 240$ & 130 & 4500 & MSS \\
\hline 2452689.577 & $4290 \pm 480$ & 110 & 4500 & MSS \\
\hline 2452805.343 & $5180 \pm 470$ & 180 & 4500 & MSS \\
\hline 2452807.392 & $6230 \pm 360$ & 140 & 4500 & MSS \\
\hline 2452812.372 & $1460 \pm 290$ & 130 & 4500 & MSS \\
\hline 2452830.403 & $5840 \pm 500$ & 200 & 4500 & MSS \\
\hline 2452831.435 & $6660 \pm 430$ & 200 & 4500 & MSS \\
\hline 2452832.496 & $7170 \pm 390$ & 170 & 4500 & MSS \\
\hline 2452834.431 & $4710 \pm 510$ & 170 & 4500 & MSS \\
\hline 2452835.380 & $3610 \pm 290$ & 240 & 4500 & MSS \\
\hline 2452838.390 & $4900 \pm 500$ & 200 & 6560 & MSS \\
\hline 2452840.433 & $6660 \pm 500$ & 240 & 6560 & MSS \\
\hline 2453666.163 & $6980 \pm 240$ & 240 & 4500 & MSS \\
\hline \multicolumn{5}{|c|}{ Intensity spectra } \\
\hline HJD & $\left\langle B_{\mathrm{s}}\right\rangle(\mathrm{kG})$ & $S / N$ & $R$ & Instrument \\
\hline 2453591.362 & $17.1 \pm 0.4$ & 200 & 43300 & NES \\
\hline 2453599.357 & $17.4 \pm 0.5$ & 140 & 37600 & NES \\
\hline 2453600.263 & $17.3 \pm 0.4$ & 250 & 37600 & NES \\
\hline 2453600.575 & $18.0 \pm 0.5$ & 60 & 115000 & HARPS \\
\hline
\end{tabular}

was determined from the relative shift of the profiles of metal spectral lines recorded in the left and right circularly polarized spectra. Positions of spectral lines were determined using the Gaussian approximation of line profiles. Strong magnetic field of HD 178892 produces a noticeable partial Zeeman splitting even in low resolution MSS spectra. To exclude the influence of this effect on the longitudinal field measurements, we selected only lines which were symmetrical relative to the line centre and did not show Zeeman splitting. This explains a difference of our results with the previous studies based on the same observations (El'kin et al. 2003; Kudryavtsev et al. 2004), where the field was determined using an express procedure without careful selection of spectral lines. Therefore, magnetic field measurements presented here are more accurate. The log of spectropolarimetric observations and resulting $\left\langle B_{\mathrm{z}}\right\rangle$ measurements are summarised in Table 1.

High-resolution intensity spectra were obtained for HD 178892 in August 2005 using the cross-dispersed Nasmyth Echelle Spectrometer (NES, Panchuk et al. 1999) installed at the SAO 6-m telescope and using the HARPS spectrograph at the 3.6-m telescope of ESO. The NES spectra, covering the wavelength region $\lambda \lambda$ 4687-6140 $\AA$ at the resolving power $R=37600-43300$, were reduced with the help of the IDL-based package REDUCE developed by Piskunov \& Valenti (2002). One HARPS observation in the $\lambda \lambda$ 3780-6910 $\AA$ wavelength domain was obtained at $R=115000$ and was reduced using the standard pipeline procedure available for this instrument. Table 1 gives information on our high-resolution spectroscopic observations of HD 178892.
One photometric measurement of HD 178892 was made in the seven passbands of the Geneva system (Golay 1980) with the refurbished photoelectric photometer P7 (Burnet \& Rufener 1979) attached to the 1.2-m Mercator telescope at La Palma. The resulting colours and reddening-free parameters are given in Table 2. These data will soon be included in the up-to-date database maintained by Burki et al. (2005), which complements the Geneva Photometric Catalogue of Rufener (1988).

\section{Fundamental parameters}

Since the Geneva reddening-free parameter $Y$ is significantly lower than zero, the star is clearly cooler than $\sim 9000 \mathrm{~K}$ (Cramer $\&$ Maeder 1980). Thus, one can use the $B 2-G$ index to estimate the effective temperature through the calibration of Hauck \& North (1993). Neglecting interstellar reddening, this calibration gives $T_{\text {eff }} \gtrsim 7130 \mathrm{~K}$. However, the Hipparcos parallax of this star being $3.70 \pm 1.18$ mas, its distance is about $270 \mathrm{pc}$, so that reddening may be significant. According to the maps of Lucke (1978) and given the low galactic latitude $\left(b=+2.78^{\circ}\right)$, the colour excess $E(B-V)$ may be as large as $\sim 0.2$, leading to $T_{\text {eff }} \sim 8440 \mathrm{~K}$. On the other hand, the $0.11 \AA$ equivalent width of the interstellar $\mathrm{Na}$ D2 line indicates a considerably lower colour excess, $E(B-V)=0.03$ (Munari \& Zwitter 1997), and results in $T_{\text {eff }} \sim 7300 \mathrm{~K}$. An intermediate value $E(B-V)=0.1$ would lead to $T_{\text {eff }} \approx 7700 \mathrm{~K}$. Although uncertainty in the reddening does not allow us to make an accurate temperature estimate from the photometry, analysis of the hydrogen line profiles can decrease substantially the allowed temperature range. The observed $\mathrm{H} \alpha$ and $\mathrm{H} \beta$ profiles provide more strict limits for the effective temperature: $7500 \mathrm{~K} \leq T_{\text {eff }} \leq 8000 \mathrm{~K}$.

The luminosity of HD 178892 is more difficult to determine because of the low precision of the Hipparcos parallax. All that can be said, though with much caution, is that HD 178892 appears to be a slightly evolved star with $M \approx 1.8-2.4 M_{\odot}$. Assuming the most probable effective temperature of $7700 \mathrm{~K}$ and neglecting the Lutz-Kelker correction on the luminosity, interpolation in the evolutionary tracks of Schaller et al. (1992) gives $\log g \approx 3.79 \pm 0.24$. This is smaller than the surface gravity of an unevolved star (for which $\log g \sim 4.3$ ), but remains consistent with it within slightly more than $2 \sigma$. With the given uncertainty of the parallax and the effective temperature it is difficult to make more definite conclusion about the evolutionary status of HD 178892.

\section{Rotation and magnetic field}

Based on $\left\langle B_{\mathrm{z}}\right\rangle$ measurements Kudryavtsev et al. (2004) estimated the rotation period of HD 178892 to be $P=8.27 \pm$ $0.08 \mathrm{~d}$. They did not find any periodic variability of the star in the Hipparcos epoch photometry (ESA 1997). In addition to the Hipparcos mission, HD 178892 was observed by the All Sky Automated Survey (ASAS, Pojmanski 2002) and by the Northern Sky Variability Survey (NSVS, Woźniak et al. 2004). The latter photometry is made in a wide spectral band with the effective wavelengths of the $R$ filter, while the ASAS data (150 points) are obtained in the $V$ system over the time interval of 888 days. A search for periodicity using the Fourier 
Table 2. Geneva colour indices of HD 178892, based on a single measurement. Errors are typically about 0.006 mag on each colour. This corresponds to the quality of the measurement $P=2$ on the scale defined by Rufener (1988).

\begin{tabular}{ccccccccccc}
\hline \hline HJD & $V$ & {$[U]$} & {$[V]$} & B1 & B2 & V1 & G & B2-G & X & Y \\
\hline 2453605.512 & 8.926 & 1.396 & 0.568 & 0.968 & 1.391 & 1.283 & 1.671 & -0.280 & 1.154 & -0.230 \\
\hline
\end{tabular}

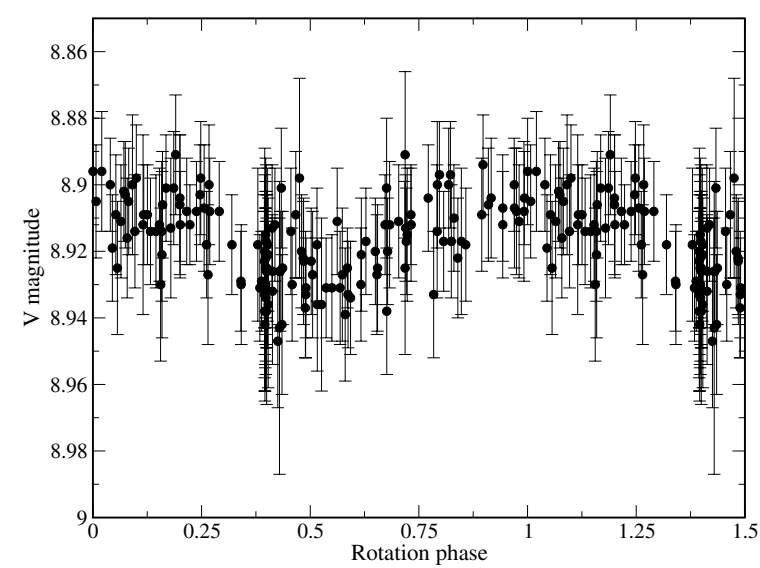

Fig. 1. Photometric variation of HD 178892 with a 8.2478 d period.

Transform technique realized in the PERIOD04 software package (Lenz 2004) does not reveal any variation in the NSVS data, whereas a clear signal at $P=8.25 \mathrm{~d}$ appears in the ASAS photometry. A refined period search with the help of leastsquares IDL routines resulted in the following ephemeris for HD 178892:

$\operatorname{HJD}\left(V_{\max }\right)=2452708.562+8.2478(76) \cdot E$.

No other statistically significant peaks were found in the power spectrum as well as in the residuals after subtracting the main frequency. Figure 1 shows the ASAS $V$-band variation phased with $P=8.2478 \mathrm{~d}$. The variability of the longitudinal magnetic field is illustrated in Fig. 2. From the $\left\langle B_{\mathrm{z}}\right\rangle$ curve we estimate the minimum possible polar field strength to be $B_{\mathrm{p}} \approx 23 \mathrm{kG}$, assuming a dipolar field topology. The corresponding inclination angle is $i=37^{\circ}$, which coincides with $i=36 \pm 15^{\circ}$ derived from the usual oblique rotator relation using $P=8.2478 \mathrm{~d}$, $T_{\text {eff }}=7700 \pm 300 \mathrm{~K}$, and the Hipparcos parallax. The magnetic obliquity $\beta$ is $24-55^{\circ}$ for this permitted range of $i$, whereas $\left\langle B_{\mathrm{s}}\right\rangle$ lies in between 15.1 and $21.1 \mathrm{kG}$ depending on the exact values of the angles and rotation phase.

Due to substantial magnetic broadening, it is rather difficult to derive an accurate rotational velocity. Spectral lines with negligible splitting, e.g., Fe I 5434 and $5576 \AA$ are weak and blended. The fit to the Fe II 4491, $4508 \AA$ and Cr II $4634 \AA$ lines with Landé factors $0.4-0.5$, along with the Fe I $5434 \AA$ line allows us to estimate $v_{\mathrm{e}} \sin i=9 \pm 1 \mathrm{~km} \mathrm{~s}^{-1}$ as the most probable value of the projected rotational velocity.

HD 178892 is a definite spectral variable (Fig. 3), but we do not expect to see significant variations in high-resolution spectra because they all are obtained near the maximum of $\left\langle B_{\mathrm{z}}\right\rangle$ in the phase interval $0.040-0.150$. Figure 4 displays part of the spectrum at $5018 \AA$, where a triplet-like splitting of the Fe II $5018.45 \AA$ line is clearly detected in high-resolution spectra.

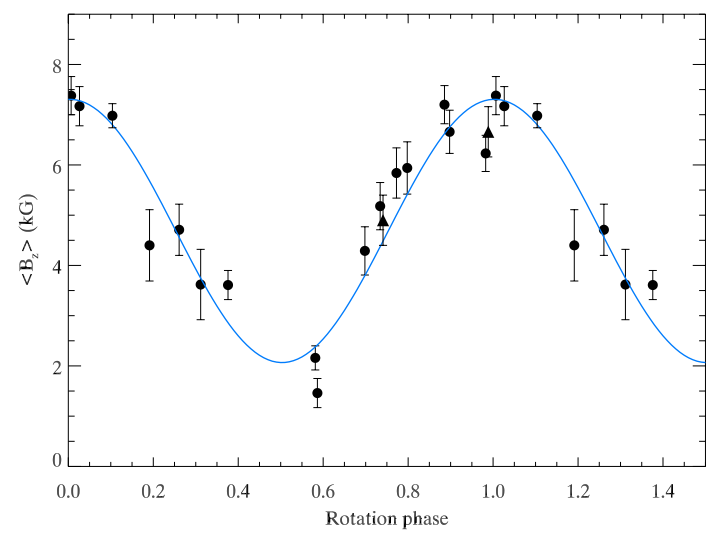

Fig. 2. Longitudinal field measurements for HD 178892 phased with $P=8.2478$ d. Symbols show metal line (circles) and $\mathrm{H} \alpha$ (triangles) $\left\langle B_{\mathrm{z}}\right\rangle$ determinations. Solid line shows $\left\langle B_{\mathrm{z}}\right\rangle$ variation expected for the dipolar field topology with $i=36^{\circ}$ (assumed), $B_{\mathrm{p}}=23 \mathrm{kG}$, and $\beta=37^{\circ}$ (derived from $\left\langle B_{\mathrm{z}}\right\rangle$ observations).

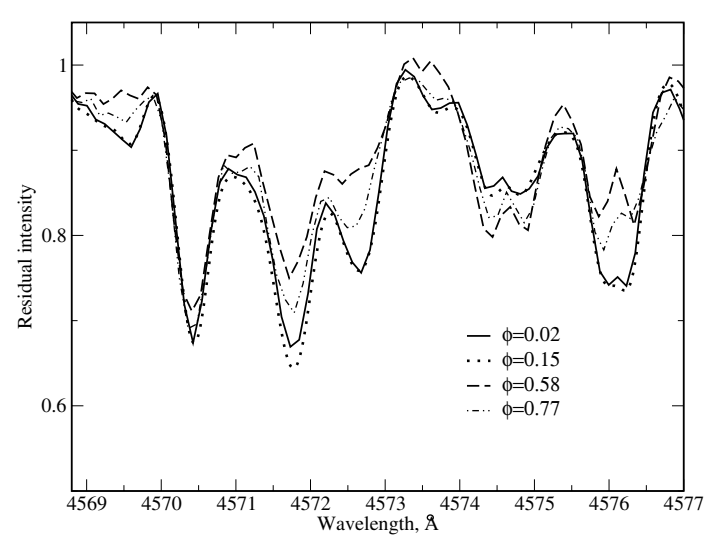

Fig. 3. Spectral variations of HD 178892 (MSS observations).

Gaussian approximation for the resolved Zeeman components allow us to measure magnetic splitting and deduce the magnetic field modulus $\left\langle B_{\mathrm{s}}\right\rangle$, ranging from $17.1 \pm 0.4 \mathrm{kG}$ (1st NES spectrum) to $18.0 \pm 0.5 \mathrm{kG}$ (HARPS spectrum). The dipolar field geometry derived from the fit to the $\left\langle B_{z}\right\rangle$ data (see Fig. 2) predicts a $18.0-18.5 \mathrm{kG}$ field modulus for the corresponding rotational phase interval. Thus, given uncertainty in mutual phasing of the spectropolarimetry and high-resolution intensity observations, as well as possible deviation of magnetic topology from a centred dipole, we conclude that $\left\langle B_{\mathrm{z}}\right\rangle$ and $\left\langle B_{\mathrm{s}}\right\rangle$ measurements agree with each other fairly well.

A synthetic spectrum calculated using SYNTHMAG code (Piskunov 1999) with $\left\langle B_{\mathrm{S}}\right\rangle=17.5 \mathrm{kG}$ is compared with observations in Fig. 4. 


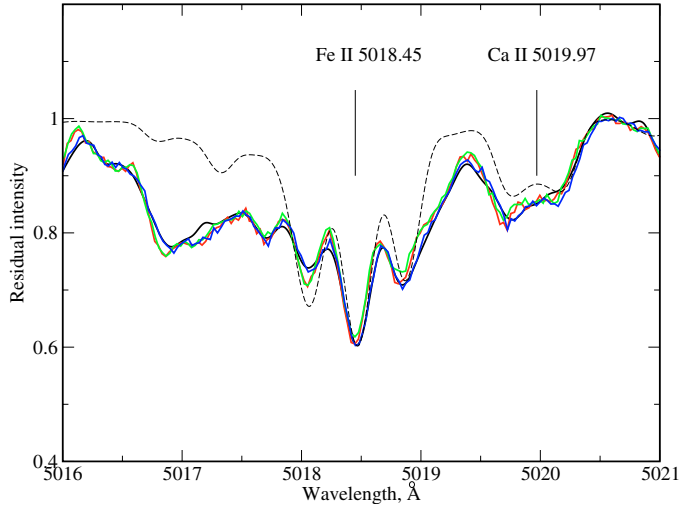

Fig. 4. The spectrum of HD 178892 in the vicinity of the Fe II $5018.45 \AA$ line. Solid lines show high-resolution observations, whereas dashed line represents synthetic spectrum calculation with $\left\langle B_{\mathrm{s}}\right\rangle=17.5 \mathrm{kG}$.

Table 3. Element abundances in the atmosphere of HD 178892 derived using $n$ lines. Error estimates are given in paranthesis. The last column contains new solar abundances from Asplund et al. (2005).

\begin{tabular}{llrr|llrr}
\hline \hline Ion & $\log \left(N_{\text {el }} / N_{\text {tot }}\right)$ & $n$ & Sun & Ion & $\log \left(N_{\text {el }} / N_{\text {tot }}\right)$ & $n$ & Sun \\
\hline Mg I & -5.20 to -5.80 & 3 & -4.51 & Fe I & -5.20 to -5.50 & 5 & -4.59 \\
Mg II & -5.30 & 1 & -4.51 & Fe II & $-5.00(10)$ & 5 & -4.59 \\
Si I & $-3.70:$ & 1 & -4.53 & Ba II & -10.30 & 2 & -9.87 \\
Si II & $-3.60(15)$ & 4 & -4.53 & Pr II & $-8.66(40)$ & 3 & -11.33 \\
Ca I & -6.00 to -7.70 & 4 & -5.73 & Pr III & $-7.44(34)$ & 6 & -11.33 \\
Ca II & -5.60 to -7.70 & 2 & -5.73 & Nd II & $-8.05(23)$ & 7 & -10.59 \\
Sc II & $-8.50:$ & 1 & -8.99 & Nd III & $-6.84(33)$ & 10 & -10.59 \\
Cr I & -5.50 to -6.00 & 6 & -6.40 & Eu II & $-8.35(25)$ & 4 & -11.52 \\
Cr II & -5.00 to -5.50 & 6 & -6.40 & Tb III & $-7.38(32)$ & 4 & -11.76 \\
\hline
\end{tabular}

\section{Abundances}

Abundances in the atmosphere of HD 178892 were estimated from high-resolution spectra for a model with the parameters $T_{\text {eff }}=7700 \mathrm{~K}, \log g=4.0$, and $\left\langle B_{\mathrm{s}}\right\rangle=17.5 \mathrm{kG}$ using the SYNTHMAG code for magnetic spectrum synthesis. The results of this analysis are presented in Table 3. Being derived for the phases near magnetic maximum, they are preliminary because we did not consider the surface chemical inhomogeneity. The atmosphere of HD 178892 is deficient in $\mathrm{Mg}$, Ba, and some Fepeak elements $(\mathrm{Ca}, \mathrm{Fe}, \mathrm{Ni})$. This is a characteristic signature of cool roAp stars with slightly lower temperatures. Abundances of the rare-earth elements (REE) in HD 178892 are also similar to those found in cool roAp stars. In particular, a strong PrNd anomaly (significant violation of the ionization equilibrium, see Ryabchikova et al. 2004) is present. Extremely large dispersion in abundance obtained from the resonance and subordinate $\mathrm{Ca}$ I and Ca II lines clearly indicate the presence of $\mathrm{Ca}$ stratification. Effective temperature variation in the limits provided by the hydrogen line profiles does not result in substantial changes of the derived chemical abundances.

\section{Conclusions}

HD 178892 is a new cool photometric, spectroscopic, and magnetic variable. Unusually short rotation period of $8.25 \mathrm{~d}$ and a very large magnetic field make this star a perfect target for detailed study of the field geometry and surface chemical inhomogeneities using magnetic Doppler imaging (Piskunov \& Kochukhov 2002). The position of HD 178892 in the H-R diagram amongst roAp stars, as well as the observed abundance anomalies, make this star a suitable target for the search of rapid oscillations. New photometric and high-resolution spectropolarimetric observations are needed to improve determination of the rotation period and to study in details the surface magnetic field and abundance distributions.

Acknowledgements. We are very grateful to N. Samus for his helpful advises concerning the automated photometric surveys. We also warmly thank Prof. G. Burki and an anonymous observer for the photometric measurement in the Geneva system. This research was supported by RFBR grant 03-02-16342a, by Russian President's Foundation for young scientists (MK-1424.2005.2), by Austrian Science Fonds (FWF-P17580N2) and by the Swiss National Science Foundation.

\section{References}

Asplund, M., Grevesse, N., \& Sauval, A. J. 2005, ASP Conf. Ser., 336, 25

Babcock, H. W. 1960, ApJ, 132, 521

Burnet, M., \& Rufener, F. 1979, A\&A, 74, 54

Burki, G. et al. 2005, GENEVA photometric database, http://obswww. unige.ch/gcpd/ph13.html

Chuntonov, G. A. 1997, in Stellar magnetic fields, ed. Yu. V. Glagolevskij, \& I. I. Romanyuk, Moscow, RAS, 229

Cramer, N., \& Maeder, A. 1980, A\&A, 88,135

El'kin, V. G., Kudryavtsev, D. O., \& Romanyuk, I. I. 2003, Astron. Lett., 29, 400

ESA 1997, The Hipparcos Catalogue, ESA SP-1200

Golay, M. 1980, Vistas Astron., 24, 141

Hauck, B., \& North, P. 1993, A\&A, 269, 403

Kudryavtsev, D. O. 2000, in Magnetic fields of chemically peculiar and related stars, ed. Yu. V. Glagolevskij, \& I. I. Romanyuk, Moscow, RAS, 84

Kudryavtsev, D. O., Romanyuk, I. I., \& Elkin, V. G. 2004, in Magnetic Stars 2003, ed. Yu. V. Glagolevskij, D. O. Kudryavtsev, \& I. I. Romanyuk, Moscow, RAS, 93

Lenz, P. 2004, Commun. Asteroseismol., 144, 41

Lucke, P. B. 1978, A\&A, 64, 367

Hubrig, S., Nesvacil, N., Schöller, M., et al. 1997, A\&A, 440, L37

Munari, U., \& Zwitter, T. 1997, A\&A, 318, 269

Najdenov, I. D., \& Chuntonov, G. A. 1976, Soobshch. Spec. Astrofiz. Obs., 16, 63

Panchuk, V. E., Klochkova, V. G., \& Najdenov, I. D. 1999, Preprint of the Spec. Astrophys. Obs., 135

Piskunov, N. E. 1999, in 2nd International Workshop on Solar Polarization, ed. K. Nagendra, \& J. Stenflo (Kluwer Acad. Publ. ASSL), 243, 515

Piskunov, N., \& Kochukhov, O. 2002, A\&A, 381, 736

Piskunov, N. E., \& Valenti, J. A. 2002, A\&A, 385, 1095

Pojmanski, G. 2002, Acta Astronomica, 52, 397

Ryabchikova, T., Nesvacil, N., Weiss, W. W., et al. 2004, A\&A, 423, 705

Rufener, F. 1988, GENEVA Photometric Catalogue, 4th edition, Observatoire de Genève

Schaller, G., Schaerer, D., Meynet, G., \& Maeder, A. 1992, A\&AS, 96, 269

Woźniak, P. R., Vestrand, W. T., Akerlof, C. W., et al. 2004, AJ, 127, 2436 